\title{
MODELLING AND PROFILING STUDENT DESIGNERS' COGNITIVE COMPETENCIES IN COMPUTER-AIDED DESIGN
}

\author{
Clay, John (1); \\ Li, Xingang (2); \\ Rahman, Molla Hafizur (2); \\ Zabelina, Darya (1); \\ Xie, Charles (3); \\ Sha, Zhenghui (2) \\ 1: Department of Psychological Science, University of Arkansas; \\ 2: Department of Mechanical Engineering, University of Arkansas; \\ 3: Institute for Future Intelligence
}

\begin{abstract}
There are three approaches to studying designers - through their cognitive profile, design behaviors, and design artifacts (e.g., quality). However, past work has rarely considered all three data domains together. Here we introduce and describe a framework for a comprehensive approach to engineering design, and discuss how the insights may benefit engineering design research and education. To demonstrate the proposed framework, we conducted an empirical study with a solar energy system design problem. Forty-six engineering students engaged in a week-long computer-aided design challenge that assessed their design behavior and artifacts, and completed a set of psychological tests to measure cognitive competencies. Using a machine learning approach consisting of k-means, hierarchical, and spectral clustering, designers were grouped by similarities on the psychological tests. Significant differences were revealed between designer groups in their sequential design behavior, suggesting that a designer's cognitive profile is related to how they engage in the design process.
\end{abstract}

Keywords: Design cognition, Computer Aided Design (CAD), Human behaviour in design

\section{Contact:}

Sha, Zhenghui

University of Arkansas

Mechanical Engineering

United States of America

zsha@uark.edu

Cite this article: Clay, J., Li, X., Rahman, M. H., Zabelina, D., Xie, C., Sha, Z. (2021) 'Modelling and Profiling Student Designers' Cognitive Competencies in Computer-Aided Design', in Proceedings of the International Conference on Engineering Design (ICED21), Gothenburg, Sweden, 16-20 August 2021. DOI:10.1017/pds.2021.477 


\section{INTRODUCTION}

For an engineer to be successful it is important they can design and understand complex systems. As technology has progressed, the systems that engineers design and interact with have become increasingly elaborate (Frank, 2000), and as technology continues to advance, the skills necessary for success will likely grow in complexity. Thus, engineers need to learn these skills to be sufficiently prepared to enter the workplace. However, universities educating young engineers may have difficulties preparing them for their careers - one of the reasons is that researchers have been unable to decide which skills and traits are relevant to success in systems design.

Systems design is a complex cognitive skill that is executed in the mind of the designer and thus potentially influenced by many aspects of a designer's cognitive profile, including cognitive skills, personality traits, previous experiences, etc. Not all aspects of cognition play a role in design - the exact mental traits that drive design, along with the extent to which they are important, has been the topic of a great deal of research. The study of the relevant cognitive traits falls under design thinking the cognition that designers engage in while solving a design problem (Dym et al., 2006).

Closely related to design thinking is systems thinking, presently defined as all cognitive processes and traits relevant to systems design. There are many models of systems thinking, few of which are in agreement with each other on the relevant processes and traits. In the present study, we leverage Moti Frank's Capacity for Engineering Systems Thinking (CEST) model. The CEST model proposes a cognitive profile of a successful systems designer based on sixteen traits and skills (i.e., cognitive competencies) which were observed in senior systems designers who had demonstrated their skill through accomplished industry careers (2010). However, the model is not concerned with the quantitative measurement of these competencies. To address this, Greene and Papalambros (2016) mapped the CEST competencies to psychological constructs. We further this approach and apply psychometrics to objectively measure the competencies that comprise the cognitive profile of a successful systems designer.

Design is not a single task, but rather a unique problem-solving situation that is iteratively solved by designers. This sequential cycle is known as the design process. Previous research has examined the number of stages within the design process and what each stage consists of, although no consensus yet exists regarding the number or content of stages. The present study models the design process with the Function-Behavior-Structure (FBS) ontology (Gero, 1990). One of the biggest challenges in modelling the design process is that the stages that designers work through are often domain or problem-specific, thus making a generalizable design process model difficult to extract. The FBS model was built with an emphasis on domain-generalizability, and each stage consists of general design behaviors.

Previous design research generally collects data in one of three domains: cognitive profile, design process behavior, and a description of the artifact. These types of data each offer unique insights into the nature of design, the designer's cognitive competencies, and how they are related; however, they are rarely analyzed together. To obtain an in-depth understanding of how people carry out design and which cognitive competencies make up the cognitive profile of a successful systems designer, we must consider the relationships between the three domains of data that designers offer. With this approach we can extract the full cognitive profile of a successful designer of engineering systems and define the cognitive competencies.

Many benefits can be gleaned from illuminating the cognitive profile of successful designers. For example, the research could be used to guide the feedback or instructions that designers receive while engaging in a design task. As the relationships between designers' cognitive profile, process behaviors, and design artifacts are made clear, behavior from one domain may be used to predict or guide the behavior in another. If the designers' cognitive profile is detectable through design process behaviors, by analysing these behaviors, the designers' thinking patterns can be predicted and guided through curated artificial intelligence (AI) assistance and recommendation.

Studying design process behavior may be done with computer-aided design (CAD) software that logs each process behavior. The software may become a personalized pedagogical tool if it possesses the functionality to predict the cognitive profile of a designer with process behaviors and artifacts. For example, consider a designer with process behavior recognized by CAD software as highly predictive of a poorly designed artifact. Explicitly guiding process behavior can be intrusive and does not offer many lessons on design - guiding the designer's cognition would an effective way to change their behavior. For example, if it is found that these design process behaviors are similar to the ones 
frequently displayed by designers with low creative ability, then an intervention may be made on the designer. It should be noted that psychological literature offers many techniques for fostering creativity (for a review, see Scott et al. 2004).

The current paper proposes a framework that models designers based on the data they offer for their cognitive profile, design process behavior, and designed artifact. We used the machine-learning approach to group them by their similarities. Once designers are grouped by data in one domain (here, their cognitive profile), tests of differences may then be performed between the groups on the behaviors they show in the other domains (here, their design process behavior). After explaining the framework in further detail, an empirical study is presented to demonstrate its application.

\section{METHODOLOGY}

This section discusses the general approach taken by the framework and will follow from top to bottom the flow of Figure 1. The figure begins with the three domains of collected data, followed by the data cleaning process, the partitioning of designers, the created designer groups, and lastly, the tests of difference on designer behavior. For data analysis, each designer was modelled through two methods. Firstly, designers were defined by a transition matrix derived from their design process behavior with a discrete-time Markov chain (DTMC). Secondly, designers were partitioned according to the similarities they showed in their cognitive data through three different cluster analysis algorithms. The variation of the information method was then used to determine the best clustering. The method and details described here are similar to that of Rahman et al. (2018). Note that in the present study, data on design artifacts were not addressed yet.

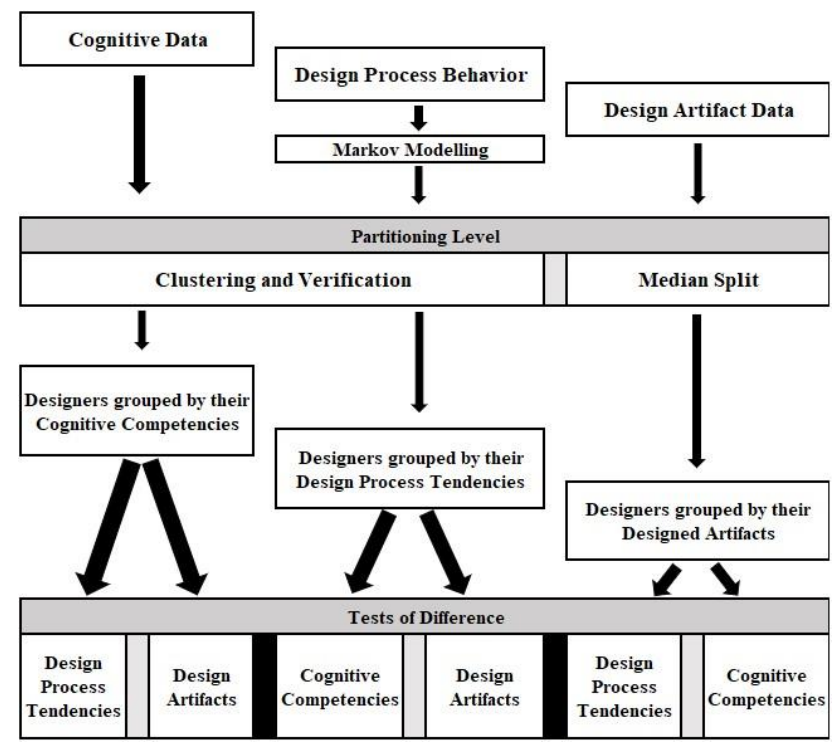

Figure 1. The described framework.

\subsection{Data Collection}

The present study employs validated psychometric tests that are widely used within the literature on neuroscience and psychology to measure the competencies hypothesized to be relevant to systems thinking. A description of each competency and the test used to measure it can be found in Section 2.2.

Design process behavior and design artifact data may be obtained through CAD software. In this study, we adopt Energy3D (Xie et al., 2018) - A computer-aided solar systems design software, which allows users to design buildings while interacting with solar energy and the environment to test the energy efficiency of their designs. By setting goals for students, researchers obtain a design artifact for analysis. Additionally, Energy3D sequentially logs fine-grained design action data.

\subsection{Statistical Modelling}

Designers can display hundreds of design process behaviors, and thus it is important for researchers to classify process behaviors with a design ontology model. We began by classifying each designer action along with one of five design process stages. We had intended to use all eight stages in the FBS model 
(Gero, 1990); however, we found that designers did not use actions in four of the stages (Documentation, Evaluation, Reformulation 1, Reformulation 3). It is likely that the design problem (described in Section 2.1) did not offer participants the opportunity to engage in all eight design process stages.

The stages that design process behavior was classified by were: Formulation (adding a component to the design), Analysis (checking the energy efficiency of a design), Synthesis (editing any component), and Reformulation 2 (removing any component). Additionally, designers were displaying behaviors that did not fit within the stages of the FBS model. These unclassified behaviors were similar in that they each allowed the designer to test the parameters of the virtual environment that they were interacting with. For example, a participant may observe differences in shadow casting patterns by changing between months. An Environmental Check design process stage was added to account for these behaviors. See Table 1 for the design stages, examples of the actions that fell within each stage, and the total number of actions observed across all designers in the study described in the following section.

Table 1. The design process stages and examples of the corresponding actions.

\begin{tabular}{|l|c|r|}
\hline Design Process Stage & Design Behaviors & No. of Actions \\
\hline Formulation & "Add Solar Panel" & 9,954 \\
\hline Analysis & $\begin{array}{c}\text { "Daily Energy Analysis," "Annual Energy } \\
\text { Analysis" }\end{array}$ & 13,029 \\
\hline Synthesis & "Rotate Solar Panel," "Change Tilt Angle" & 71,558 \\
\hline Reformulation 2 & "Remove Solar Panel" & 7,690 \\
\hline Environmental Check & "Change Time and Date," "Animate Sun" & 42,091 \\
\hline
\end{tabular}

Once designers' process behavior was classified, a Markov chain was used to model the probabilities that designers showed for transitioning between design process stages. Markov models have been well employed in design research (for example, McComb et al., 2017). In the present study, each designer was defined across a 5 x 5 matrix containing the probabilities they showed for each possible stage-tostage transition. The probability that a designer transitioned from Stage A to Stage B was equal to the number of times they followed an action in Stage A with an action in Stage B divided by the number of overall actions they made in Stage A. With 5 stages to transition between, design process behavior was defined across 25 variables.

Following this, designers were partitioned by the similarities in their responses to the psychometric tests. Designers were grouped through three different cluster analysis algorithms (k-means, hierarchical, spectral), after which the variation of information method was used to determine single best clustering. So that clusterings may be compared, the elbow plot generated for the k-means algorithm was used to guide the other algorithms, wherein for each $k$ in the range suggested by the elbow plot, three different clusterings were performed. The generated elbow plot suggested a $k$ range of six to nine, offering twelve potential solutions for grouping designers.

K-means and hierarchical clustering algorithms are commonly used clustering methods, and we avoid discussing them in much depth. Spectral clustering has recently proliferated throughout clustering literature due to simplicity and the performance in relation to traditional algorithms, and the main strength is in the restraint it shows in making strong assumptions on the subsequent iterations of groupings; this makes it ideal for large data sets (Von Luxburg, 2007). This method uses eigenvalues to define observations and then compares them using similarity matrices before grouping.

To determine the best of the twelve potential solutions, the variation of information technique was used. This method compares the amount of information that is gained and lost between two clusterings of the same data set by using the total within-cluster sum of squares to measure compactness (Meilă, 2003). A value for each possible pairing of clusterings is calculated, and any pairing that falls within the top $25 \%$ is considered efficient. The solution that offers the highest number of efficient pairings is deemed the best answer.

Once the optimal clustering is revealed, tests of difference may then be performed to investigate whether these groups differ in the data domain that they were not clustered by. To demonstrate, below we describe an empirical study that groups designers based on their cognitive competencies and then tests for differences in their design process behaviors. 


\section{THE EMPIRICAL STUDY}

\subsection{Methods}

\subsubsection{Participants}

Data were collected from 49 students (38 male, 11 female; 39 undergraduate and ten graduate) at the University of Arkansas, with an average age of $22.91(S D=4.38)$ who participated in a week-long systems design challenge. The researchers did not expect any demographic variables to play a role in the subsequent analyses - this information is included for contextual and replication purposes. Recruitment was done through flyers, advertisements in a local university newspaper, and the offer of extra credit in several undergraduate classes. Potential participants were informed that they would receive $\$ 500, \$ 250$, and $\$ 100$ prizes for the $1^{\text {st }}, 2^{\text {nd }}$, and $3^{\text {rd }}$ place winners, respectively. The study was approved by the university's Institutional Review Board, and all participants provided informed consent prior to joining the contest.

\subsubsection{Procedure}

Data collection was conducted in two sessions. Session 1 was conducted in person, and Session 2 was conducted virtually. The researchers had intended to conduct both sessions in person, but as the COVID-19 outbreak forced the end to all in-person data collection, Session 2 data collection was conducted online. A series of t-tests were performed between the normalized scores to the psychological tests between Sessions 1 and 2. No significant differences were found, all $p$ 's $>.34$.

Participants began the design challenge by attending an introduction presentation containing all of the information required to complete the challenge, after which they were given seven full days to work on the design task. After they finished the task, participants were administered the psychological tests. The challenge ended with an award ceremony the following week. In Session 1 (in-person data collection), participants completed the psychological tests on university laptops and with a researcher present. In Session 2 (online data collection), participants completed the tests via Qualtrics - the online questionnaire service, on their own devices and with no researcher present.

\subsubsection{The Design Challenge}

To elicit meaningful design process behaviors from designers, a complex systems design problem was developed. Designers completed the challenge through Energy3D. Energy3D allows users to build, edit, and analyse the efficiency of green structures and is growing in popularity as an effective tool in engineering design research (for an overview, see Rahman et al., 2019).

Through internal testing, constraints and goals were fine-tuned to create an intricate design problem consisting of many interrelated variables for the designers to manipulate in the design process. The challenge was created to both encourage designers to engage in systems thinking and to give them a large design space to explore as they worked through the design process. Designers were told that the problem was open-ended and that there was no single correct solution. They were encouraged to work in an iterative process throughout the week to create a design that met the goals and the constraints.
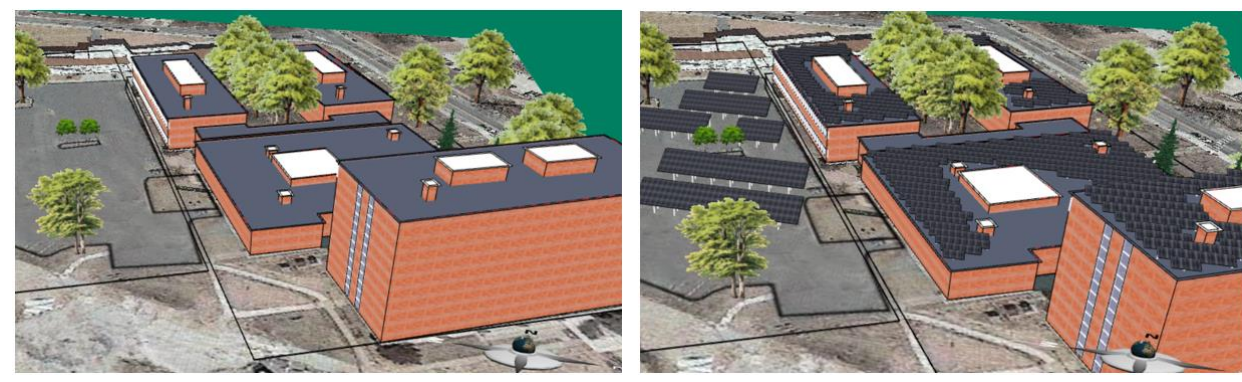

Figure 2. Left panel: the model in which participants began their design.

Right panel: An example of a submitted design that met the goals and constraints.

Participants received a design statement that outlined a project to bring a solar energy system to their local university campus. Using the area on the rooftop and in the adjacent parking lot of a student housing complex, participants were called to compete against each other in designing a solution. 
Figure 2 shows the empty model that designers were given (left panel), and an example of a submitted design (right panel). The only objects which participants used in their designs were solar panels, although the panels had many variables over which the designers had control. Participants manipulated solar panel height, width, length, tilt angle, model, placement, and arrangement. Each of these variables interacted with each other (for example, the optimal tilt angle changed depending on the placement of the panel) in a way that forced designers to engage in systems thinking.

Participants were given three main goals to achieve: an annual energy output of $1,000,000 \mathrm{kWh}$, a payback period of 10 years, with an overall budget of $\$ 1,900,000$. In addition to the goals, participants were also challenged with several constraints. For example, designers had to arrange solar panels while maintaining a minimum distance between them, and they were required to choose one from three panel model options (see Table 2 for an overview of the goals and constraints).

Table 2. The goals and constraints which designers had to consider.

\begin{tabular}{|l|c|r|}
\hline \multicolumn{2}{|c|}{ Variable } & \multicolumn{1}{|c|}{ Benchmark } \\
\hline Goals & Annual Energy Output & $1,000,000 \mathrm{kWh}$ \\
\cline { 2 - 3 } & Payback Period & 10 years \\
\cline { 2 - 3 } & Budget & $\$ 1,900,000$ \\
\hline Constraints & Solar Panel Model & Choose 1 of 3 options \\
\cline { 2 - 3 } & Solar Panel Height & $\geq 3.5 \mathrm{~m}$ \\
\cline { 2 - 3 } & Solar Panel Width & $5.25 \mathrm{~m}-6 \mathrm{~m}$ \\
\cline { 2 - 3 } & Panel Placement (overall) & Panel edges must not overlap \\
\cline { 2 - 3 } & Panel Placement (in the parking lot) & $\geq 7 \mathrm{~m}$ from the nearest panel \\
\hline
\end{tabular}

\subsection{Measures}

\subsubsection{Creativity}

Creativity is a widely studied construct within many fields, including engineering design. There is little argument of whether creativity plays a role in design (Starkey et al., 2019). Current research focuses on how creativity is related to design, and which measures the best capture the creative cognitive processes that contribute to the cognitive profile of a successful designer. Creativity is a multidimensional construct that engages many cognitive processes (Kühn et al., 2014). In the present study, we measure divergent thinking, which allows the designer to engage in idea generation.

In the first session, participants completed the Abbreviated Torrance Test of Creative Thinking (ATTA; Goff and Torrance, 2002). The ATTA allows the researchers to capture several aspects of creativity, including fluency (number of generated ideas) and originality (the novelty of ideas). For the second session, the Alternate Uses Task (AUT; Guilford, 1967) was used and was also scored for fluency and originality. The ATTA measures both verbal and figural creativity, but as the AUT measures only verbal creativity, we observed only the verbal creativity scores from the ATTA.

\subsubsection{Personality}

Designers were measured across one of the personality traits from the Big Five personality inventory (BFI; McCrae and John, 1992), openness to experience. The BFI is a widely used and validated model of personality, and quantifies personality across five constructs. We assessed the openness to experience trait as it is most directly evident in the CEST model (Frank, 2010). Openness to experience captures CEST's description of the curiosity and ability to consider multiple perspectives, as well as an openness to new ideas, feelings, and working outside of the status quo found in designers. Additionally, openness to experience has shown to be positively related to various measures of creativity (Feist, 1998). Across 10 prompts, participants rated their personality on a 5-point Likert scale ranging from 1 (Very Inaccurate) to 5 (Very Accurate).

\subsubsection{Cognitive Ability}

Cognitive ability is a broad psychological term for general reasoning abilities and has previously been shown to predict academic performance (for an overview, Brandt et al., 2020). We used the International Cognitive Ability Resource was used (ICAR; Condon and Revelle, 2014) to measure cognitive ability. The ICAR was designed to capture general reasoning and logical thinking skills and 
has shown positive correlations with both scores on the ACT and SAT (Frey and Detterman, 2004; Koenig et al., 2008). The ICAR is optimized and free to all researchers and has been widely used in psychology (Dworak et al., 2020). The 16 items on the ICAR are traditionally split into four categories: Letter and Number Series, Matrix Reasoning, Verbal Reasoning, and Three-Dimensional Rotation. Designer general cognitive ability is quantified by the sum of correctly answered items.

\subsubsection{Working Memory}

Working memory allows one to store, consider, and manipulate information (Baddeley, 1992) and likely plays a role in any task where one must deal with large amounts of information at once. For the first session, we used the Keep Track test of working memory (adapted from Miyake et al., 2000). Participants were asked to selectively remember predetermined categories of neutral words (animals, relatives, etc.) out of a larger set of words. For the second session, participants completed a publicly available audio-visual n-back test (Jaeggi et al., 2008). In the n-back test participants are given a list of stimuli, and then are asked to recall prior items on the list. Participants were asked to recall an item that was presented a certain number (n) of items prior, and the total number of correct answers indicated working memory capacity.

\subsubsection{Imagination}

Imagination is a complex, multifaceted construct, and in the present study, we used the Four-Factor Imagination Scale (FFIS; Zabelina and Condon, 2019) to characterize individual differences in the imagination of designers. The FFIS consists of 26 items and measures imagination across four subconstructs: frequency (time spent imagining), complexity (detail of imagination), emotional valence (positive or negative imagination), and directedness (goal-oriented imagination). Participants answered each item on a 6-point Likert Scale ranging from 1 (very inaccurate) to 6 (very accurate), and their answers within the constructs were averaged for the four sub-scales scores. The FFIS has shown good internal consistency ( $\alpha=.87$ overall, $\alpha=.76-.93$ for the subscales), and displays good convergent and discriminant validity.

\subsubsection{Design Process Behavior}

Every behavior designers exhibited across the design challenge was logged sequentially. Participants showed a total of 144,322 actions $(M=3,069.97, S E=351.23)$, and 37 unique actions.

\subsection{Results}

Design process behavior was defined across a 5 x 5 matrix for the probabilities designers showed for transitioning between design process stages, after which designers were grouped according to similarities in their cognitive profile. The elbow plot suggested that the $k$ value that best captured the data was between six and nine, and each designer was clustered along three algorithms for the suggested values. Variation of information showed that the most efficient algorithm was K-means when $k$ was equal to eight.

Cognitive data for 3 of the 49 participants were discarded because these participants misunderstood test instructions; thus, $n=46$. Except for Group Five, membership was split modestly fair between clusters. The $n$ of each designer group along with the average normalized scores for the cognitive competencies can be seen in Table 3 (note: scores for the FFIS are not included in the table below).

Table 3. Mean (Standard Error) for the normalized scores in each cluster group. $D T=$ Divergent Thinking

\begin{tabular}{|l|c|c|c|c|c|c|}
\hline Group & $\mathrm{n}$ & DT - Fluency & DT - Orig. & Openness & Cog. Ability & Wrk. Mem. \\
\hline One & 4 & $0.554(.337)$ & $0.651(.269)$ & $-1.427(.200)$ & $-0.229(.297)$ & $0.691(.615)$ \\
\hline Two & 5 & $-0.639(.337)$ & $-1.098(.317)$ & $-1.292(.371)$ & $-1.659(.270)$ & $-0.685(.456)$ \\
\hline Three & 8 & $-0.527(.331)$ & $-0.653(.222)$ & $0.915(.128)$ & $-0.689(.237)$ & $-0.617(.264)$ \\
\hline Four & 5 & $0.033(.294)$ & $0.195(.172)$ & $-0.142(.408)$ & $0.352(.451)$ & $-0.188(.390)$ \\
\hline Five & 10 & $1.368(2.18)$ & $0.767(.344)$ & $0.702(.196)$ & $0.509(.294)$ & $0.491(.228)$ \\
\hline Six & 3 & $-0.901(.357)$ & $-1.163(.221)$ & $-0.244(.278)$ & $1.106(.181)$ & $1.608(.466)$ \\
\hline Seven & 6 & $-0.205(.189)$ & $-0.244(.228)$ & $-0.116(.107)$ & $0.216(.375)$ & $-0.272(.176)$ \\
\hline Eight & 5 & $-0.557(.225)$ & $1.093(.264)$ & $0.625(.205)$ & $0.352(.213)$ & $-0.015(.413)$ \\
\hline
\end{tabular}


A series of one-way between-subjects analyses of variance (ANOVAs) were conducted to test for differences in the probabilities that designers showed for transitioning between design process stages among the created designer groups. Twenty-five ANOVAs were performed on the 25 possible transitions. Significant differences were found between group behaviors in four transitions (Table 4).

Table 4. Significant differences between designer groups in process behavior.

\begin{tabular}{|l|c|c|c|}
\hline Design Process Behavior & $F$ & $p$ & $\eta^{2}$ \\
\hline Synthesis to Synthesis & $F(7,38)=2.859$ & .017 & .251 \\
\hline Synthesis to Reform. 2 & 2.554 & .029 & .328 \\
\hline Synthesis to Analysis & 2.489 & .034 & .320 \\
\hline Env. Check to Formulation & 2.331 & .045 & .306 \\
\hline
\end{tabular}

Tukey's post hoc comparisons showed that participants in Group Four $(M=.721, S E=.080)$ were significantly less likely to conduct sequential Synthesis actions than participants in Groups Two $(M=$ $.785, S E=.039), p=.021$, Five $(M=.785, S E=.024), p=.034$ and $\operatorname{Six}(M=.838, S E=.029), p=.032$. Group Three $(M=.041, S E=.006)$ was significantly more likely to transition from Synthesis to Reformulation 2 than Group Eight $(M=.016, S E=.004), p=.048$.

Group Four $(M=.051, S E=.099)$ transitioned from the Synthesis to Analysis stage significantly more often than Group Three $(M=.226, S E=.013), p=.042$.

Finally, post hoc comparisons revealed no significant differences between any two groups on the Environmental Check to Formulation transition.

\section{DISCUSSION}

The current study grouped designers based on their cognitive competencies and found significant differences in the probabilities designer groups showed for transitioning between design process stages. Three of the four significant tests were found in the transition probabilities following the Synthesis stage, suggesting that the cognitive competencies measured in our sample of designers were most relevant to the process behaviors in and after the Synthesis stage.

In the Synthesis stage, designers engage in revisional behaviors to edit the structure of their design. Within the context of the design problem, designers engaged in Synthesis process behaviors when they edited any aspect of the solar panels, such as the length, width, height, or tilt angle. Of the 144,322 process behaviors, 71,588 (49.58\%) were Synthesis actions. With almost half of the overall actions being classified in one stage, it is possible that our sample was not adequately powered to detect all present effects.

Three of the five possible stage transitions following Synthesis were used significantly different between the designer groups, but only one of the non-Synthesis transitions was significant. The measured cognitive competencies were predominantly related to editing behaviors and mostly unrelated to behaviors in other stages of the design process. Despite differences in cognitive profile, our sample showed similar design behaviors in certain stages. For example, designers showed an overall probability of $84.91 \%$ to transition from one Environmental Check stage behavior to another. However, their cognitive competencies were significantly related to the behavior they showed when revising and editing their designs. These results suggest that the measured cognitive competencies are related to how designers sequentially behave while revising their designs, and that through observing these editing behaviors we may begin to grasp the cognitive profile of designers.

\subsection{Limitations}

There were several limitations to our study. First, data collected occurred in two sessions, six months apart. While we do not expect the time passed to have altered the design skills of the general population, the onset of the COVID-19 pandemic between the two sessions may have had a cognitive impact in our sample. Design is a cognitive task, and literature is growing quickly on the negative psychological effects of the pandemic and related issues (for example, Kontoangelos et al., 2020). Additionally, data collection for the two sessions was done in different formats (in-person vs. online). The framework that we describe here is also not without limitations. First, as $k$ increases, the required $n$ to reach sufficient statistical power increases as well. The pilot study of our framework suggested that the best clustering algorithm had a $k$ of 8 . With a sample size of 46 , our investigations were likely 
underpowered. Additionally, the relatively large number of ANOVA's conducted on design process behaviors likely increased our chances of encountering both Type I and Type II errors.

\subsection{Future Directions}

There are many potential future directions for this research approach. First, only a subset of the data collected in the study described here was presented in the results. Designer partitions based on the other domains, and other tests of differences may be conducted further. For example, data on designed artifacts were not collected in enough detail to warrant machine learning techniques and were not addressed in the current paper. The framework that is introduced here was described in general so that researchers may adapt it for future investigations.

Additionally, it is unlikely that the cognitive competencies assessed in the present study are the only competencies related to systems design. Design is an exceedingly complex process and one that is not yet fully understood. In future studies, designers should be tested across a wider set of cognitive constructs. In addition to explicit cognitive skills, other psychological traits should be examined as well. For example, one's background (socio-economic status, education), social skills (willingness to work in teams, agreeableness, communication skills), emotions (emotional intelligence, general affect during design), and motivation all likely play a role in systems design success, and should be considered in future work.

In sum, the presented approach on the relationships between designers' cognition and design process behavior creates the potential for additional research on how designers' thoughts may be guided as they engage in design. This has the potential to offer many pedagogical benefits, as those who are learning design may be able to obtain immediate feedback and personalized lessons to guide their systems thinking and design behavior.

\section{CONCLUSION}

The present study introduces, describes, and demonstrates a framework for grouping designers by their cognitive competencies. Forty-six designers who engaged in a week-long computer-aided design task and completed a set of psychological tests were clustered according to their cognitive competencies. Tests of differences found significant differences in design process behaviors between designer groups of different cognitive profiles. These findings show that the sequential design process behavior of designers is related to a subset of the cognitive competencies that are important to success in engineering systems design.

\section{ACKNOWLEDGMENTS}

The authors would like to gratefully acknowledge the financial support from the U.S. National Science Foundation (NSF) via grants \#1842588, \#1503196, and \#1918847. Any opinions, findings, and conclusions expressed in this publication or presentation are those of the authors and do not necessarily reflect the view of the NSF.

\section{REFERENCES}

Baddeley, A. (1992), “Working memory”, Science, 255(5044), pp. 556-559.

Brandt, N. D., Lechner, C. M., Tetzner, J., \& Rammstedt, B. (2020), "Personality, cognitive ability, and academic performance: Differential associations across school subjects and school tracks", Journal of personality, 88(2), pp. 249-265.

Condon, D. M., \& Revelle, W. (2014), “The International Cognitive Ability Resource: Development and initial validation of a public-domain measure", Intelligence, 43, pp. 52-64.

Dworak, E. M., Revelle, W., Doebler, P., \& Condon, D. M. (2021), "Using the International Cognitive Ability Resource as an open source tool to explore individual differences in cognitive ability", Personality and Individual Differences, 169, 109906.

Dym, C. L., Agogino, A. M., Eris, O., Frey, D. D., \& Leifer, L. J. (2006), “Engineering design thinking, teaching, and learning”, IEEE Engineering Management Review, 34(1), pp. 65-65.

Feist, G. J. (1998), “A meta-analysis of personality in scientific and artistic creativity”, Personality and social psychology review, 2(4), pp. 290-309.

Frank, M. (2000), “Engineering systems thinking and systems thinking”, Systems Engineering, 3(3), pp. 163-168. 
Frank, M. (2010), “Assessing the interest for systems engineering positions and other engineering positions' required capacity for engineering systems thinking (CEST)", Systems Engineering, 13(2), pp. 161-174.

Frey, M. C., \& Detterman, D. K. (2004), "Scholastic assessment or g? The relationship between the scholastic assessment test and general cognitive ability", Psychological science, 15(6), pp. 373-378.

Gero, J. S. (1990), "Design prototypes: a knowledge representation schema for design”, AI magazine, 11(4), pp. 26-26.

Goff, K., Torrance, E.P. (2002), “Abbreviated Torrance Test for Adults manual”, Bensenville, IL: Scholastic Testing Service.

Greene, M. T., \& Papalambros, P. Y. (2016, March), “A cognitive framework for engineering systems thinking”, 2016 Conference on Systems Engineering Research pp. 1-7.

Guilford, J.P. (1967), “The Nature of Human Intelligence”, New York: McGraw-Hil1.

Jaeggi, S. M., Buschkuehl, M., Jonides, J., \& Perrig, W. J. (2008), "Improving fluid intelligence with training on working memory", Proceedings of the National Academy of Sciences, 105(19), pp. 6829-6833.

Koenig, K. A., Frey, M. C., \& Detterman, D. K. (2008), “ACT and general cognitive ability”, Intelligence, 36(2), pp. 153-160.

Kontoangelos, K., Economou, M., \& Papageorgiou, C. (2020), "Mental health effects of COVID-19 pandemia: a review of clinical and psychological traits", Psychiatry Investigation, 17(6), 491.

Kühn, S., Ritter, S. M., Müller, B. C., Van Baaren, R. B., Brass, M., \& Dijksterhuis, A. (2014), “The importance of the default mode network in creativity-A structural MRI study", The Journal of Creative Behavior, 48(2), 152-163.

McComb, C., Cagan, J., \& Kotovsky, K. (2017), “Capturing human sequence-learning abilities in configuration design tasks through markov chains”, Journal of Mechanical Design, 139(9): 091101 pp. 1-12.

McCrae R. R., John O. P.; John (1992), “An introduction to the Five-Factor Model and its applications”, Journal of Personality. 60 (2): pp. 175-215. https://dx.doi.org/10.1111/j.1467-6494.1992.tb00970.

Meilă, M. (2003), "Comparing clusterings by the variation of information”, Learning theory and kernel machines, pp. 173-187.

Miyake, A., Friedman, N. P., Emerson, M. J., Witzki, A. H., Howerter, A., \& Wager, T. D. (2000), “The unity and diversity of executive functions and their contributions to complex "frontal lobe" tasks: A latent variable analysis", Cognitive psychology, 41(1), pp. 49-100.

Rahman, M. H., Gashler, M., Xie, C., \& Sha, Z. (2018, August), “Automatic Clustering of Sequential Design Behaviors", International Design Engineering Technical Conferences and Computers and Information in Engineering Conference (Vol. 51739, pp. V01BT02A041). American Society of Mechanical Engineers.

Rahman, M. H., Schimpf, C., Xie, C., \& Sha, Z. (2019), “A Computer-Aided Design Based Research Platform for Design Thinking Studies”, Journal of Mechanical Design, 141(12): 121102 pp. 1-12.

Scott, G., Leritz, L.E., Mumford, M.D. (2004) "Types of creativity training: Approaches and their effectiveness", The Journal of Creative Behavior, 38(3), pp. 149-179.

Starkey, E. M., Hunter, S. T., \& Miller, S. R. (2019), “Are creativity and self-efficacy at odds? an exploration in variations of product dissection in engineering education", Journal of Mechanical Design, 141(1).

Von Luxburg, U. (2007), “A tutorial on spectral clustering”, Statistics and computing, 17(4), pp. 395-416.

Xie, C., Schimpf, C., Chao, J., Nourian, S., \& Massicotte, J. (2018), "Learning and teaching engineering design through modeling and simulation on a CAD platform", Computer Applications in Engineering Education, 26(4), pp. 824-840.

Zabelina, D. L., \& Condon, D. M. (2019), “The Four-Factor Imagination Scale (FFIS): A measure for assessing frequency, complexity, emotional valence, and directedness of imagination”, Psychological Research, pp. 1-13. 